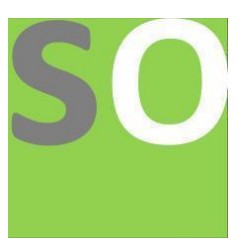

Article title: Using the Membership Table Method (MTM) to Determine the Validity of Categorical Syllogisms Authors: Ricardo E. Monge[1], Osvaldo Skliar[2], Sherry Gapper[3]

Affiliations: Universidad Cenfotec, Costa Rica[1], Universidad Nacional, Costa Rica[2]

Orcid ids: 0000-0002-4321-5410[1], 0000-0002-8321-3858[2], 0000-0003-4920-6977[3]

Contact e-mail: ricardo@acl.ac.cr

License information: This work has been published open access under Creative Commons Attribution License $\mathrm{http}: / / c r e a t i v e c o m m o n s . o r g / l i c e n s e s / b y / 4.0 /$, which permits unrestricted use, distribution, and reproduction in any medium, provided the original work is properly cited. Conditions, terms of use and publishing policy can be found at https://www.scienceopen.com/.

Preprint statement: This article is a preprint and has not been peer-reviewed, under consideration and submitted to ScienceOpen Preprints for open peer review.

DOI: 10.14293/S2199-1006.1.SOR-.PPIG4XQ.v1

Preprint first posted online: 13 October 2021

Keywords: propositional calculus, set theory, categorical syllogisms, truth tables, membership tables, membership table method 


\title{
Using the Membership Table Method (MTM) to Determine the Validity of Categorical Syllogisms
}

\author{
Osvaldo Skliar* Sherry Gapper ${ }^{\dagger} \quad$ Ricardo E. Monge ${ }^{\ddagger}$
}

October 11, 2021

\begin{abstract}
A presentation is provided of a method - the Membership Table Method (MTM) - to determine the validity of categorical syllogisms. This method makes it possible for each syllogism to be assigned a specific set. If this set is equal to the universal set $\mathbb{U}$, then the categorical syllogism considered is valid, and if that set is not equal to $\mathbb{U}$, then that categorical syllogism is not valid. In other words, any categorical syllogism is valid if and only if its respective set, according to the MTM, is equal to the universal set $\mathbb{U}$. The conclusion of a valid categorical syllogism whose premises are true is true.
\end{abstract}

Keywords: propositional calculus, set theory, categorical syllogisms, truth tables, membership tables, membership table method

Mathematics Subject Classification 2020: 03B05, 97E60, 03E20, 03B10, 97E30

\footnotetext{
*Universidad Nacional, Costa Rica. E-mail: osvaldoskliar@gmail.com. https://orcid.org/0000-0002-8321-3858.

$\dagger$ Universidad Nacional, Costa Rica. E-mail: sherry.gapper.morrow@una.ac.cr. https://orcid.org/0000-0003-4920-6977.

$\ddagger$ Universidad CENFOTEC, Costa Rica. E-mail: rmonge@ucenfotec.ac.cr. https://orcid.org/0000-0002-4321-5410.
} 


\section{Introduction}

From historical and educational standpoints, forms of reasoning known as syllogisms, especially categorical syllogisms, have been relevant in the development of logic.

The objective of this article is to present a method to determine the validity of categorical syllogisms: the membership table method (MTM). It can be applied more simply and systematically than other pre-existing methods. Up until now one of the most commonly used approaches to determine the validity of categorical syllogisms has been that of using diverse types of diagrams [1], [2], [3], [4], [5], and [6]. A previous contribution by the authors of this article, with that objective, can be classified within that approach [7].

A clear understanding of this article requires only a basic knowledge of the propositional calculus of classical logic and of set theory. For an introduction to classical logic, one may consult, for example: [8], [9], [10], [11], and [12]. On set theory, one may consult, for example, [13], [14], [15], and [16].

\section{A Correspondence between Operations of Pro- positional Calculus and Operations of Set The- ory}

Propositional variables - that is, variables that can be replaced with propositions - are usually referred to as $p, q, r$, etc. Given that in other articles related to this one the letter $p$ will be used to refer to a probability and diverse probabilities will be denominated $p_{1}, p_{2}, p_{3}$, etc., to prevent confusion, in this article these propositional variables will be symbolized as follows: $q_{1}, q_{2}, q_{3}$, etc. (with some exceptions as specified in section 5). In addition, taking advantage of a "license" used by a number of authors, $q_{1}, q_{2}, q_{3}$, etc. will be referred to as "propositions". Thus, statements such as " $q_{1}$ is true" and " $q_{2}$ is false" should be interpreted respectively in the following way: "Admit that $q_{1}$ has been replaced with a true proposition" and "Admit that $q_{2}$ has been replaced with a false proposition". If a sole proposition is considered, the subscript can be eliminated and the proposition symbolized as $q$.

In set theory, the universal set, or the universe of discourse, to which all the elements that may be considered when referring to a given topic belong, will be symbolized as $\mathbb{U}$.

The diverse sets characterized within the framework of the universal set $\mathbb{U}$ will be denominated $C_{1}, C_{2}, C_{3}$, etc. (with some exceptions to be specified in section 6). If only one of those sets is considered, the subscript can be eliminated and the set symbolized as $C$.

The (monadic) logical connective of negation in propositional calculus will be symbolized by a horizontal bar above the negated proposition. Thus, the symbol $\bar{q}$ - that is, not $q$ - will represent the negation of that proposition $q$.

All the elements of the set $\mathbb{U}$ considered that do not belong to $C$ belong to 
the complement of the set $C$. The complement of $C$ is symbolized as $\stackrel{+}{C}$. In this article the symbol + corresponding to the operator of complementation of a set is placed above its operand. This operand is the set $C$ on which that operator acts, thus generating the complement of $C$ (that is, $\vec{C}$ ), which is another set.

Given that all of the elements which may be considered when dealing with a particular topic belong to the set $\mathbb{U}$, no element belongs to the complement of $\mathbb{U}$, known as the empty set. It is symbolized as $\varnothing$.

The relation between the universal set $\mathbb{U}$ and the corresponding empty set can be expressed by the following equality:

$$
\overrightarrow{\mathbb{U}}=\varnothing
$$

Given that a) all the elements belonging to $\mathbb{U}$ that do not belong to $C$ belong to the complement of any set $C$ and that b) no element of the corresponding $\mathbb{U}$ belongs to the set $\varnothing$, it is obvious that

$$
\stackrel{+}{\varnothing}=\mathbb{U}
$$

Given that because of (2), $\bar{\varnothing}$ and $\mathbb{U}$ are the same set, their complements must also be equal:

$$
\stackrel{+}{\varnothing}=\stackrel{+}{\mathbb{U}}
$$

If, in (3), $\stackrel{+}{\mathbb{U}}$ is replaced with $\varnothing$, admissible given the equality (1), the following equation is obtained:

$$
\stackrel{+}{\varnothing}=\varnothing
$$

In addition, given that because of (1), $\stackrel{+}{\mathbb{U}}$ and $\varnothing$ are the same set, their complements will also be equal:

$$
\stackrel{+}{+}=\stackrel{+}{\varnothing}
$$

According to (2), $\stackrel{+}{\varnothing}$ can be replaced in (5) with $\mathbb{U}$. Thus the following equation is obtained:

$$
\stackrel{+}{\mathbb{U}}=\mathbb{U}
$$

In general, the complement of the complement of any set $C$ is equal to $C$ :

$$
\stackrel{+}{+}=C
$$

The truth tables (a) of $q, \bar{q}$ and $\overline{\bar{q}}$, and the membership tables (b) of $C, \stackrel{+}{C}$

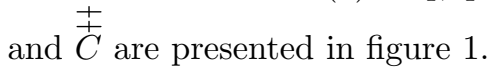




\begin{tabular}{l||l||l}
$q$ & $\bar{q}$ & $\overline{\bar{q}}$ \\
\hline 0 & 1 & 0 \\
1 & 0 & 1
\end{tabular}

(a) Truth tables of $\bar{q}$ (that is, of the negation of $q$ ) and of $\overline{\bar{q}}$ (that is, of the negation of the negation of $q$, or of the "double negation" of $q$ ).

\begin{tabular}{c||c||c}
$C$ & $\stackrel{+}{C}$ & $\stackrel{+}{C}$ \\
\hline 0 & 1 & 0 \\
1 & 0 & 1
\end{tabular}

(b) Membership tables of $\stackrel{+}{C}$ (that is, of the complement of $C$ ) and of $\stackrel{+}{C}$ (that is, of the complement of the complement of $C$ ).

Figure 1: a) Truth tables of $\bar{q}$ and $\overline{\bar{q}}$, and b) membership tables of $\vec{C}$ and $\stackrel{\vec{C}}{\text {. }}$

In figure $1 \mathrm{a}$, the presence of a 0 in the column corresponding to a proposition, such as $q$, means that the proposition is considered false. The presence of a 1 in the column corresponding to a proposition means that the proposition is considered true. Classical logic is bivalent, in the sense that there are only two possible truth values for any proposition: It is true or it is false. In that bivalent logic, the negation of any true proposition is a false proposition, and the negation of any false proposition is a true proposition.

The first row of the truth tables represented in figure 1a is composed of the numerical sequence $0,1,0$. It should be interpreted in the following way: If the proposition $q$ is false, then its negation $\bar{q}$ is true and the negation of $\bar{q}$ (that is, $\overline{\bar{q}})$ is true.

Note in figure 1a that the columns corresponding to $q$ and $\overline{\bar{q}}$ are identical: asserting $q$ is equivalent to asserting $\overline{\bar{q}}$.

In figure $1 \mathrm{~b}$, the set $C$ mentioned above is any set characterized within the framework of any universal set $\mathbb{U}$; that is, any element belonging to $C$ is an element belonging to that $\mathbb{U}$.

In figure $1 \mathrm{~b}$, the presence of a 0 in a column corresponding to a set, such as $C$, means the supposition that any element belonging to $\mathbb{U}$ does not belong to that set. The presence of a 1 in the column corresponding to a set means the supposition that any element belonging to $\mathbb{U}$ does belong to that set. In classic set theory, if any element of $\mathbb{U}$ belongs to $C$ then that element does not belong to $\stackrel{+}{C}$, and if that element of $\mathbb{U}$ does not belong to $C$ then that element does belong to $\vec{C}$.

The first row of the membership tables in figure $1 \mathrm{~b}$ is composed of the numerical sequence $0,1,0$. It should be interpreted as follows: If any element of $\mathbb{U}$ (that is, belonging to $\mathbb{U}$ ) does not belong to $C$, then that element does belong to the complement of $C$ (i.e., $\bar{C}$ ) and does not belong to the complement of $\stackrel{+}{C}$ (i.e., $\stackrel{+}{\vec{C}}$ ). 
The second row of the membership tables in figure $1 \mathrm{~b}$ is composed of the numerical sequence 1, 0, 1. It should be interpreted as follows: If any element of $\mathbb{U}$ belongs to $C$, then that element does not belong to the complement of $C$ (i.e., $\stackrel{+}{C}$ ) and does belong to the complement of $\stackrel{+}{C}$ (i.e., $\stackrel{+}{C}$ ).

Note that the columns corresponding to $C$ and to $\stackrel{+}{C}$ are identical: $C=\overline{\vec{C}}$, as seen in (7); the double operation of complementation of a set results in the set $C$ itself.

The first and the second rows of the truth tables in figure 1a are the same, respectively, as the first and second rows of the membership tables in figure $1 \mathrm{~b}$. That makes it possible to establish a) the correspondence of the proposition $q$ with the set $C, \mathrm{~b}$ ) the correspondence of the proposition $\bar{q}$ with the set $\vec{C}, \mathrm{c}$ ) the correspondence of the proposition $\overline{\bar{q}}$ with the set $\stackrel{\vec{C}}{\mathrm{C}}$, and d) the correspondence of the connective of negation in propositional calculus with the operator of complementation in set theory.

The logical connective of conjunction of two propositions is symbolized as $\wedge$. The proposition $q_{1} \wedge q_{2}$ - the conjunction of the propositions $q_{1}$ and $q_{2}$ - is read as: " $q_{1}$ and $q_{2}$ ".

The operator of the intersection of two sets is symbolized as $\cap$. The set which is the intersection of the sets $C_{1}$ and $C_{2}$ - two sets characterized within the framework of some universal set $\mathbb{U}$ - is symbolized as $C_{1} \cap C_{2}$.

Figure 2 presents a) the truth table of the proposition $q_{1} \wedge q_{2}$, and b) the membership table of the set $C_{1} \cap C_{2}$.

\begin{tabular}{c|c||c}
$q_{1}$ & $q_{2}$ & $q_{1} \wedge q_{2}$ \\
\hline 0 & 0 & 0 \\
0 & 1 & 0 \\
1 & 0 & 0 \\
1 & 1 & 1
\end{tabular}

(a) Truth table of the proposition $q_{1} \wedge q_{2}$.

\begin{tabular}{c|c||c}
$C_{1}$ & $C_{2}$ & $C_{1} \cap C_{2}$ \\
\hline 0 & 0 & 0 \\
0 & 1 & 0 \\
1 & 0 & 0 \\
1 & 1 & 1
\end{tabular}

(b) Membership table of the set $C_{1} \cap C_{2}$.

Figure 2: a) Truth table of $q_{1} \wedge q_{2}$; and b) membership table of $C_{1} \cap C_{2}$.

In the truth table of $q_{1} \wedge q_{2}$ (in figure 2a), it is seen that only when both $q_{1}$ and $q_{2}$ are true, is $q_{1} \wedge q_{2}$ also true. This case corresponds to the fourth row in that truth table, which is composed of the numerical sequence $1,1,1$; if and only if $q_{1}$ and $q_{2}$ are true, is $q_{1} \wedge q_{2}$ also true. 
In the membership table $C_{1} \cap C_{2}$ (in figure $2 \mathrm{~b}$ ), it is seen that only when any element of $\mathbb{U}$ belongs both to $C_{1}$ and to $C_{2}$, does that element belong to $C_{1} \cap C_{2}$. This case corresponds to the fourth row of that membership table, which is composed of the numerical sequence 1, 1, 1; if and only if any element of $\mathbb{U}$ belongs both to $C_{1}$ and to $C_{2}$, does that element also belong to $C_{1} \cap C_{2}$.

Note, in figure 2, the equality of the first, second, third and fourth rows, respectively, in figure $2 \mathrm{a}$ and figure $2 \mathrm{~b}$. This makes it possible to establish a correspondence between a) the propositions $q_{1}$ and $q_{2}$ and the sets $C_{1}$ and $C_{2}$, respectively; b) the proposition $q_{1} \wedge q_{2}$ (the conjunction of $q_{1}$ and $q_{2}$ ) and the set $C_{1} \cap C_{2}$ (the set which is the intersection of $C_{1}$ and $C_{2}$ ); and c) the logical connective of conjunction in propositional calculus $(\wedge)$ and the operator of intersection in set theory $(\cap)$.

The logical connective of disjunction of two propositions will be symbolized as $\vee$. The proposition $q_{1} \vee q_{2}$ - the disjunction of the propositions $q_{1}$ and $q_{2}-$ is read as: " $q_{1}$ or $q_{2}$ ".

The operator of union of two sets is symbolized as $\cup$. The set which is the union of the sets $C_{1}$ and $C_{2}$ - two sets characterized within the framework of some universal set $\mathbb{U}$ - is symbolized as $C_{1} \cup C_{2}$.

Figure 3 presents a) the truth table of the proposition $q_{1} \vee q_{2}$, and b) the membership table of $C_{1} \cup C_{2}$.

\begin{tabular}{c|c||c}
$q_{1}$ & $q_{2}$ & $q_{1} \vee q_{2}$ \\
\hline 0 & 0 & 0 \\
0 & 1 & 1 \\
1 & 0 & 1 \\
1 & 1 & 1
\end{tabular}

(a) Truth table of the proposition $q_{1} \vee q_{2}$.

\begin{tabular}{c|c||c}
$C_{1}$ & $C_{2}$ & $C_{1} \cup C_{2}$ \\
\hline 0 & 0 & 0 \\
0 & 1 & 1 \\
1 & 0 & 1 \\
1 & 1 & 1
\end{tabular}

(b) Membership table of the set $C_{1} \cup C_{2}$.

Figure 3: a) Truth table of $q_{1} \vee q_{2}$; and b) membership table of $C_{1} \cup C_{2}$.

Note in the truth table in figure 3 a that only when both $q_{1}$ and $q_{2}$ are false (in the first row of that table) is $q_{1} \vee q_{2}$ false. Note also in the membership table in figure $3 \mathrm{~b}$ that only when any element of $\mathbb{U}$ belongs neither to $C_{1}$ nor to $C_{2}$ (in the first row of that table) does that element not belong to $C_{1} \cup C_{2}$.

Note that the first, second, third and fourth rows in figure $3 \mathrm{a}$ are the same, respectively, as the first, second, third and fourth rows in 3b. Therefore, a correspondence can be established between a) the propositions $q_{1}$ and $q_{2}$ and the sets $C_{1}$ and $C_{2}$, respectively; b) the proposition $q_{1} \vee q_{2}$ and the set $C_{1} \cup C_{2}$; 
and c) the logical connective of disjunction in propositional calculus and the operator of union in set theory.

The logical connective of material implication in propositional calculus will be represented as $\rightarrow$. The proposition $q_{1} \rightarrow q_{2}$ is read as: "If $q_{1}$, then $q_{2}$ ". The proposition $q_{1}$ is known as the antecedent of $q_{1} \rightarrow q_{2}$ and the proposition $q_{2}$ is known as the consequent of that proposition.

The operator of the material implication in set theory will be represented as $\mapsto$. The result of that operator acting on the ordered pair of sets $\left\{C_{1}, C_{2}\right\}$ is the set $C_{1} \mapsto C_{2}$.

The proposition $q_{2} \rightarrow q_{1}$ is read as: "If $q_{2}$, then $q_{1}$ ". The proposition $q_{2}$ is known as the antecedent of $q_{2} \rightarrow q_{1}$ and the proposition $q_{1}$ is known as the consequent of that proposition.

The result of the operator of material implication acting on the ordered pair of sets $\left\{C_{2}, C_{1}\right\}$ is equal to the set $C_{2} \mapsto C_{1}$.

Figure 4 presents a) the truth tables of the propositions $q_{1} \rightarrow q_{2}$ and $q_{2} \rightarrow q_{1}$; and b) the membership tables of the sets $C_{1} \rightarrow C_{2}$ and $C_{2} \rightarrow C_{1}$.

\begin{tabular}{c|c||c||c}
$q_{1}$ & $q_{2}$ & $q_{1} \rightarrow q_{2}$ & $q_{2} \rightarrow q_{1}$ \\
\hline 0 & 0 & 1 & 1 \\
0 & 1 & 1 & 0 \\
1 & 0 & 0 & 1 \\
1 & 1 & 1 & 1
\end{tabular}

(a) Truth tables of $q_{1} \rightarrow q_{2}$ and $q_{2} \rightarrow q_{1}$.

\begin{tabular}{c|c||c||c}
$C_{1}$ & $C_{2}$ & $C_{1} \mapsto C_{2}$ & $C_{2} \nrightarrow C_{1}$ \\
\hline 0 & 0 & 1 & 1 \\
0 & 1 & 1 & 0 \\
1 & 0 & 0 & 1 \\
1 & 1 & 1 & 1
\end{tabular}

(b) Membership tables of $C_{1} \longrightarrow C_{2}$ and $C_{2} \mapsto C_{1}$.

Figure 4: a) Truth tables of the propositions $q_{1} \rightarrow q_{2}$ and $q_{2} \rightarrow q_{1}$, and membership tables of the sets $C_{1} \mapsto C_{2}$ and $C_{2} \mapsto C_{1}$.

Note in figure 4a that the only case in which the proposition $q_{1} \rightarrow q_{2}$ is false (in the third row in the truth table of that proposition) is that in which the antecedent $q_{1}$ is true and the consequent $q_{2}$ is false. In the other three cases, $q_{1} \rightarrow q_{2}$ is true. Note also in figure $4 \mathrm{~b}$, that the only case in which any element whatsoever of the universal set $\mathbb{U}$ does not belong to the set $C_{1} \rightarrow C_{2}$ (in the third row of the membership table of that set) is that in which the element belongs to $C_{1}$ and does not belong to $C_{2}$. In the other three cases, that element does belong to $C_{1} \mapsto C_{2}$.

Note in figure 4 a that the only case in which the proposition $q_{2} \rightarrow q_{1}$ is false (in the second row in the truth table of that proposition) is that in which the antecedent $q_{2}$ is true and the consequent $q_{1}$ is false. In the other three cases, 
$q_{2} \rightarrow q_{1}$ is true. Note also in figure $4 \mathrm{~b}$ that the only case in which any element whatsoever of the universal set $\mathbb{U}$ does not belong to the set $C_{2} \rightarrow C_{1}$ (in the second row of the membership table of that set) is that in which that element belongs to $C_{2}$ and does not belong to $C_{1}$. In the other three cases, that element does belong to $C_{2} \mapsto C_{1}$.

Observe that the first, second, third and fourth rows of the truth table of $q_{1} \rightarrow q_{2}$ are the same respectively, as the first, second, third and fourth rows of the membership table of $C_{1} \rightarrow C_{2}$. Observe also that the first, second, third and fourth rows of the truth table of $q_{2} \rightarrow q_{1}$ are the same, respectively, as the first, second, third and fourth rows of the membership table of $C_{2} \rightarrow C_{1}$. Therefore, a correspondence may be established between a) the propositions $q_{1}$ and $q_{2}$ and the sets $C_{1}$ and $C_{2}$, respectively; b) the propositions $q_{1} \rightarrow q_{2}$ and $q_{2} \rightarrow q_{1}$ and the sets $C_{1} \longrightarrow C_{2}$ and $C_{2} \rightarrow C_{1}$, respectively; and c) the logical connective of material implication in propositional calculus and the operator of material implication in set theory.

The logical connective of material bi-implication, or of logical equivalence in propositional calculus, will be represented as $\longleftrightarrow$. The proposition $q_{1} \longleftrightarrow q_{2}$ is read as: "The proposition $q_{1}$ is logically equivalent to the proposition $q_{2}$ ". The operator of material bi-implication in set theory will be represented as $\longleftrightarrow$.

Figure 5 presents a) the truth table of $q_{1} \longleftrightarrow q_{2}$, and b) the membership table of the set $C_{1} \longleftrightarrow C_{2}$

\begin{tabular}{c|c||c}
$q_{1}$ & $q_{2}$ & $q_{1} \longleftrightarrow q_{2}$ \\
\hline 0 & 0 & 1 \\
0 & 1 & 0 \\
1 & 0 & 0 \\
1 & 1 & 1
\end{tabular}

(a) Truth table of $q_{1} \longleftrightarrow q_{2}$.

\begin{tabular}{c|c||c}
$C_{1}$ & $C_{2}$ & $C_{1} \longleftrightarrow C_{2}$ \\
\hline 0 & 0 & 1 \\
0 & 1 & 0 \\
1 & 0 & 0 \\
1 & 1 & 1
\end{tabular}

(b) Membership table of the set $C_{1} \longleftrightarrow C_{2}$.

Figure 5: a) Truth table of $q_{1} \longleftrightarrow q_{2}$ and b) membership table of the set $C_{1} \longleftrightarrow C_{2}$.

In figure 5a it is seen that the two cases in which the proposition $q_{1} \longleftrightarrow q_{2}$ is true are those (in the first and fourth rows of the truth table of that proposition) in which $q_{1}$ and $q_{2}$ have the same value of truth. In the case represented in the first row both $q_{1}$ and $q_{2}$ are false, and in the case represented in the fourth row both $q_{1}$ and $q_{2}$ are true.

In figure $5 \mathrm{~b}$ it is seen that the two cases in which any element whatsoever of 
the universal set $\mathbb{U}$ considered belongs to the set $C_{1} \longleftrightarrow C_{2}$ are the following: a) in the case in which the element belongs neither to $C_{1}$ nor to $C_{2}$ (represented in the first row of the membership table of that set) and b) the case in which that element belongs both to $C_{1}$ and to $C_{2}$ (represented in the fourth row of the membership table of that set).

Observe that the first, second, third and fourth rows of the truth table of $q_{1} \longleftrightarrow q_{2}$ are the same, respectively, as the first, second, third and fourth rows of the membership table of $C_{1} \longleftrightarrow C_{2}$. Thus a correspondence can be established between a) the propositions $q_{1}$ and $q_{2}$ and the sets $C_{1}$ and $C_{2}$, respectively; b) the proposition $q_{1} \longleftrightarrow q_{2}$ and the set $C_{1} \longleftrightarrow C_{2}$; and c) the logical connective of material bi-implication in propositional calculus and the operator of material bi-implication in set theory.

For propositions resulting from the use of connectives, such as $q_{1} \rightarrow q_{2}$ or $q_{3} \vee q_{4}$, it can be suitable to express them in parentheses as $\left(q_{1} \rightarrow q_{2}\right)$ and $\left(q_{3} \vee q_{4}\right)$, respectively. Thus, if a connective is used with those propositions to obtain another proposition, it is clear how that has operated. For example, $\left(q_{1} \rightarrow q_{2}\right) \rightarrow\left(q_{3} \vee q_{4}\right)$ is the proposition of a conditional nature: "If $q_{1} \rightarrow q_{2}$, then $q_{3} \vee q_{4}$ ", in which $q_{1} \rightarrow q_{2}$ is the antecedent, and $q_{3} \vee q_{4}$ is the consequent. The proposition $\left(q_{1} \rightarrow q_{2}\right) \rightarrow\left(q_{3} \vee q_{4}\right)$ has been obtained by the action of the connective of material implication on the following ordered pair of propositions $\left\{\left(q_{1} \rightarrow q_{2}\right),\left(q_{3} \vee q_{4}\right)\right\}$. Likewise, for clarity, the proposition $\left(q_{1} \rightarrow q_{2}\right) \rightarrow\left(q_{3} \vee q_{4}\right)$ can be expressed in parentheses if an operation is carried out on it and on some other proposition, by using some logical connective. Hence, for example, $\left(\left(q_{1} \rightarrow q_{2}\right) \rightarrow\left(q_{3} \vee q_{4}\right)\right) \wedge\left(q_{1} \rightarrow q_{5}\right)$ is the proposition obtained through the action of the logical connective of conjunction on the propositions $\left(q_{1} \rightarrow q_{2}\right) \rightarrow$ $\left(q_{3} \vee q_{4}\right)$ and $\left(q_{1} \rightarrow q_{5}\right)$.

Given the correspondences mentioned, a) between propositions and sets, and b) between logical connectives and set theory operators, considerations of this same type concerning the use of parentheses are valid in that theory. Therefore, the set $\left(C_{1} \rightarrow C_{2}\right) \rightarrow\left(C_{3} \cup C_{4}\right)$ corresponds to $\left(q_{1} \rightarrow q_{2}\right) \rightarrow$ $\left(q_{3} \vee q_{4}\right)$, the set $C_{1} \rightarrow C_{5}$ corresponds to the proposition $q_{1} \rightarrow q_{5}$, and the set $\left(\left(C_{1} \rightarrow C_{2}\right) \longrightarrow\left(C_{3} \cup C_{4}\right)\right) \cap\left(C_{1} \longrightarrow C_{5}\right)$ corresponds to the proposition $\left(\left(q_{1} \rightarrow q_{2}\right) \rightarrow\left(q_{3} \vee q_{4}\right)\right) \wedge\left(q_{1} \rightarrow q_{5}\right)$.

If in the logical operations carried out there are $n$ propositions $-q_{1}, q_{2}$, $\ldots, q_{n}$ - in the corresponding truth tables, there will be $2^{n}$ rows because each of those propositions can have two truth values: true or false. Each row of those tables will correspond to each possible case of different assignments for the truth values of each of those $n$ propositions. Since for each of these cases there are two possible assignments of truth value for the logical function to be specified, there are $2^{\left(2^{n}\right)}$ possible logical functions of $n$ propositions. Thus, for example, if $n=2$, there are 16 possible logical functions; if $n=3$, there are 256 possible logical functions; and if $n=4$, there are 65,536 possible logical functions.

For each of the $2^{\left(2^{n}\right)}$ logical functions of $n$ propositions, for $n=1,2,3 \ldots$, there is, according to the approach used, a function of $n$ sets, which also is a set. 


\section{Isomorphism between Each Law - Theorem or Tautology - of Propositional Calculus and the Corresponding Expression of the Univer- sal Set}

This section will provide only a) the characterizations of the main notions concerning the topic discussed; and b) three examples of isomorphism existing between the tautologies of propositional calculus and the corresponding sets (according to section 2).

If a function of $n$ propositions, for $n=1,2,3, \ldots$, is true regardless of the truth values of each of those $n$ propositions, then that function, which is also a proposition, is considered a law - or tautology - of propositional calculus. A tautology is true given its logical form, or structure.

The negation of a tautology which is a function of $n$ propositions is known as a contradiction and is false, regardless of the values of truth of each of those $n$ propositions.

The law of propositional calculus known in Latin as modus tollendo ponens - that is, "the mode that, by denying, affirms" - is stated below in (8); and the corresponding set, which is isomorphic to it according to section 2 , in (9).

$$
\begin{gathered}
\left(\left(q_{1} \vee q_{2}\right) \wedge \bar{q}_{2}\right) \rightarrow q_{1} \\
\left(\left(C_{1} \cup C_{2}\right) \cap \stackrel{+}{C}_{2}\right) \mapsto C_{1}
\end{gathered}
$$

Figure 6 presents a) the truth table of the proposition specified in (8), and b) the membership table of the corresponding set specified in (9).

\begin{tabular}{c|c|c|c|c||c}
$q_{1}$ & $q_{2}$ & $q_{1} \vee q_{2}$ & $\bar{q}_{2}$ & $\left(q_{1} \vee q_{2}\right) \wedge \bar{q}_{2}$ & $\left(\left(q_{1} \vee q_{2}\right) \wedge \bar{q}_{2}\right) \rightarrow q_{1}$ \\
\hline 0 & 0 & 0 & 1 & 0 & 1 \\
0 & 1 & 1 & 0 & 0 & 1 \\
1 & 0 & 1 & 1 & 1 & 1 \\
1 & 1 & 1 & 0 & 0 & 1
\end{tabular}

(a) Truth table of the proposition specified in (8)

\begin{tabular}{c|c|c|c|c||c}
$C_{1}$ & $C_{2}$ & $C_{1} \cup C_{2}$ & $\stackrel{C}{C}_{2}$ & $\left(C_{1} \cup C_{2}\right) \cap \stackrel{+}{C}_{2}$ & $\left(\left(C_{1} \cup C_{2}\right) \cap \stackrel{C}{C}_{2}\right) \rightarrow C_{1}$ \\
\hline 0 & 0 & 0 & 1 & 0 & 1 \\
0 & 1 & 1 & 0 & 0 & 1 \\
1 & 0 & 1 & 1 & 1 & 1 \\
1 & 1 & 1 & 0 & 0 & 1
\end{tabular}

(b) Membership table of the corresponding set, specified in (9)

Figure 6: a) Truth table of the proposition specified in (8), and b) membership table of the corresponding set, specified in (9) 
In the truth table in figure $6 \mathrm{a}$ it can be seen that the proposition $\left(\left(q_{1} \vee q_{2}\right) \wedge\right.$ $\left.\bar{q}_{2}\right) \rightarrow q_{1}$ is a tautology because it is true given its logical form; that is, it is true regardless of the truth values of $q_{1}$ and $q_{2}$.

In the membership table in $6 \mathrm{~b}$ it can be seen that for any element of the universal set $\mathbb{U}$ there are four possibilities: 1 ) that it belongs neither to $C_{1}$ nor to $C_{2}$ (as in the first row of the membership table); 2) that it does not belong to $C_{1}$ and does belong to $C_{2}$ (as in the second row of the membership table); 3 ) that it belongs to $C_{1}$ and not to $C_{2}$ (as in the third row of the membership table); and 4) that it belongs both to $C_{1}$ and to $C_{2}$ (as in the fourth row of the membership table). In each of these cases, the element of $\mathbb{U}$ considered belongs to the set $\left(\left(C_{1} \cup C_{2}\right) \cap \stackrel{+}{C}_{2}\right) \longrightarrow C_{1}$. Therefore, given that any element of $\mathbb{U}$ belongs to that set, it is inferred that the set is equal to $\mathbb{U}:\left(\left(C_{1} \cup C_{2}\right) \cap \vec{C}_{2}\right) \rightarrow C_{1}=\mathbb{U}$.

The law of propositional calculus known in Latin as modus tollendo tollens - that is, the mode that by denying, denies - is stated in (10) below; and the corresponding set, which is isomorphic to it, is stated in (11).

$$
\begin{gathered}
\left(\left(q_{1} \rightarrow q_{2}\right) \wedge \bar{q}_{2}\right) \rightarrow \bar{q}_{1} \\
\left(\left(C_{1} \longrightarrow C_{2}\right) \cap \stackrel{+}{C}_{2}\right) \longrightarrow \stackrel{+}{C}_{1}
\end{gathered}
$$

Figure 7 presents a) the truth table of the proposition specified in (10) and b) the membership table of the corresponding set, isomorphic to the proposition.

\begin{tabular}{c|c|c|c|c|c||c}
$q_{1}$ & $q_{2}$ & $q_{1} \rightarrow q_{2}$ & $\bar{q}_{2}$ & $\left(q_{1} \rightarrow q_{2}\right) \wedge \bar{q}_{2}$ & $\bar{q}_{1}$ & $\left(\left(q_{1} \rightarrow q_{2}\right) \wedge \bar{q}_{2}\right) \rightarrow \bar{q}_{1}$ \\
\hline 0 & 0 & 1 & 1 & 1 & 1 & 1 \\
0 & 1 & 1 & 0 & 0 & 1 & 1 \\
1 & 0 & 0 & 1 & 0 & 0 & 1 \\
1 & 1 & 1 & 0 & 0 & 0 & 1
\end{tabular}

(a) Truth table of the proposition specified in (10)

\begin{tabular}{c|c|c|c|c|c||c}
$C_{1}$ & $C_{2}$ & $C_{1} \rightarrow C_{2}$ & $\vec{C}_{2}$ & $\left(C_{1} \rightarrow C_{2}\right) \cap \vec{C}_{2}$ & $\vec{C}_{1}$ & $\left(\left(C_{1} \rightarrow C_{2}\right) \cap \vec{C}_{2}\right) \rightarrow \overleftrightarrow{C}_{1}$ \\
\hline 0 & 0 & 1 & 1 & 1 & 1 & 1 \\
0 & 1 & 1 & 0 & 0 & 1 & 1 \\
1 & 0 & 0 & 1 & 0 & 0 & 1 \\
1 & 1 & 1 & 0 & 0 & 0 & 1
\end{tabular}

(b) Membership table of the set specified in (11)

Figure 7: a) Truth table of the proposition specified in (10), and b) membership table of the set specified in (11)

In figure 7 it is seen a) that $\left(\left(q_{1} \rightarrow q_{2}\right) \wedge \bar{q}_{2}\right) \rightarrow \bar{q}_{1}$ is a tautology of propositional calculus, and b) that the corresponding set $\left(\left(C_{1} \longrightarrow C_{2}\right) \cap \vec{C}_{2}\right) \longrightarrow \vec{C}_{1}$, which is isomorphic to it, is equal to the universal set $\left.\mathbb{U}:\left(C_{1} \longrightarrow C_{2}\right) \cap \vec{C}_{2}\right) \longrightarrow$ $\bar{C}_{1}$. 
A law of propositional calculus - one of the laws of De Morgan - is stated in (12), and the corresponding set, which is isomorphic to it, is stated in (13) below.

$$
\begin{gathered}
\overline{\left(q_{1} \wedge q_{2}\right)} \longleftrightarrow\left(\bar{q}_{1} \vee \bar{q}_{2}\right) \\
\overline{\left(C_{1} \cap C_{2}\right)} \longleftrightarrow\left(\bar{C}_{1} \cup \stackrel{+}{C}_{2}\right)
\end{gathered}
$$

Figure 8 presents a) the truth table of the proposition specified in (12) and b) the membership table of the set specified in (13).

\begin{tabular}{c|c|c|c|c|c|c||c}
$q_{1}$ & $q_{2}$ & $q_{1} \wedge q_{2}$ & $\overline{\left(q_{1} \wedge q_{2}\right)}$ & $\bar{q}_{1}$ & $\bar{q}_{2}$ & $\left(\bar{q}_{1} \vee \bar{q}_{2}\right)$ & $\overline{\left(q_{1} \wedge q_{2}\right)} \longleftrightarrow\left(\bar{q}_{1} \vee \bar{q}_{2}\right)$ \\
\hline 0 & 0 & 0 & 1 & 1 & 1 & 1 & 1 \\
0 & 1 & 0 & 1 & 1 & 0 & 1 & 1 \\
1 & 0 & 0 & 1 & 0 & 1 & 1 & 1 \\
1 & 1 & 1 & 0 & 0 & 0 & 0 & 1
\end{tabular}

\begin{tabular}{|c|c|c|c|c|c|c|c|c|}
\hline$C_{1}$ & $C_{2}$ & $C_{1} \cap C_{2}$ & $\overline{\left(C_{1} \cap C_{2}\right)}$ & $\stackrel{+}{C}_{1}$ & $\overleftarrow{C}_{2}$ & $\left(\overleftarrow{C}_{1} \cup \bar{C}_{2}\right)$ & $\overline{\left(C_{1} \cap C_{2}\right)}$ & $\leftrightarrow\left(\overleftarrow{C}_{1} \cup \overleftarrow{C}_{2}\right)$ \\
\hline 0 & 0 & 0 & 1 & 1 & 1 & 1 & & 1 \\
\hline 0 & 1 & 0 & 1 & 1 & 0 & 1 & & 1 \\
\hline 1 & 0 & 0 & 1 & 0 & 1 & 1 & & 1 \\
\hline 1 & 1 & 1 & 0 & 0 & 0 & 0 & & 1 \\
\hline
\end{tabular}

(a) Truth table of the proposition specified in (12)

(b) Membership table of the set specified in (13)

Figure 8: a) Truth table of the proposition specified in (12), and b) membership table of the set specified in (13)

In figure 8 it is seen a) that $\left(\overline{q_{1} \wedge q_{2}}\right) \longleftrightarrow\left(\bar{q}_{1} \vee \bar{q}_{2}\right)$ is a tautology of propositional calculus and b) that the corresponding set $\left(\overline{C_{1} \cap C_{2}}\right) \longleftrightarrow\left(\stackrel{+}{C}_{1} \cup \stackrel{+}{C}_{2}\right)$, which is isomorphic to it, is equal to the universal set $\mathbb{U}:\left(\overline{C_{1} \cap C_{2}}\right) \longleftrightarrow\left(\bar{C}_{1} \cup \bar{C}_{2}\right)=$ U.

\section{Categorical Propositions and Their Corres- ponding Sets}

Categorical propositions are assertions about two sets that affirm or deny that one of those sets is totally or partially included in the other set. In this section those sets are called $C_{4}$ and $C_{5}$ respectively. Those names have been chosen for the following reason: When referring to categorical syllogisms, reasoning of a particular type, the names $C_{1}, C_{2}$ and $C_{3}$ will be used to refer systematically to certain sets which play an important role in that reasoning. To avoid confusion, 
those three names are not used in this section. Of course, instead of using $C_{4}$ and $C_{5}$, names such as $F$ and $G$ respectively could have been used.

Four examples of categorical propositions are given below:

1. All generals are brave.

2. No generals are brave.

3. Some generals are brave.

4. Some generals are not brave.

In each of the four categorical propositions, reference is made to the following two sets:

$C_{4}$ : generals; $C_{5}$ : brave persons

The following is another set of four examples of categorical propositions:

1. All philosophers are honest.

2. No philosophers are honest.

3. Some philosophers are honest.

4. Some philosophers are not honest.

The sets referred to in each of these other four categorical propositions are these:

$C_{4}$ : philosophers; $C_{5}$ : honest persons

If both sets of categorical propositions are compared, it is noted that each proposition of the first set has the same logical form as the proposition to which the same number was assigned in the second set. Thus, four types of categorical propositions can be distinguished. Any categorical proposition, given its logical form, may be ascribed to one of these four types.

The propositions assigned number 1, in both sets of four propositions, are examples of those known as affirmative universal propositions. The symbolic expression of either of them is the following:

$$
\text { All } C_{4} \text { are } C_{5} \text {. }
$$

According to the preceding proposition, if any element whatsoever of the universal set $\mathbb{U}$ considered belongs to the set $C_{4}$, then it belongs also to the set $C_{5}$. If that proposition is true, then the possibility that any element whatsoever of $\mathbb{U}$ could belong to $C_{4}$ and not also to $C_{5}$ is eliminated. According to section 2 , any element of $\mathbb{U}$ belonging to the set $C_{4} \rightarrow C_{5}$ satisfies that condition; according to the membership table of $C_{4} \longrightarrow C_{5}$, the possibility that the element could belong to $C_{4}$ and not to $C_{5}$ is eliminated.

The symbolic expression of any affirmative universal categorical proposition is stated once more below, with the corresponding set at the right. 


$$
\text { All } C_{4} \text { are } C_{5} . \quad\left(C_{4}+C_{5}\right)
$$

The propositions assigned number 2, in both sets of four propositions, are examples of those called negative universal propositions. The symbolic expression of either of them is the following:

$$
\text { No } C_{4} \text { are } C_{5} \text {. }
$$

According to the preceding proposition, if any element of the universal set $\mathbb{U}$ considered belongs to the set $C_{4}$, then it does not belong to the set $C_{5}$; that is, it also does belong to the set $\vec{C}_{5}$. If that proposition is true, then the possibility of any element whatsoever of $\mathbb{U}$ belonging to $C_{4}$, but not belonging also to $\vec{C}_{5}$, is eliminated. Any element of $\mathbb{U}$ belonging to the set $\left(C_{4} \longrightarrow \bar{C}_{5}\right)$ satisfies this condition: According to the membership table of $\left(C_{4} \rightarrow C_{5}\right)$, the possibility of that element belonging to $C_{4}$, but not belonging also to $\vec{C}_{5}$, is eliminated.

Below the symbolic expression of any negative universal categorical proposition is given once more, with the corresponding set on the right.

$$
\text { No } C_{4} \text { are } C_{5} . \quad\left(C_{4}+\overleftarrow{C}_{5}\right)
$$

The propositions assigned number 3, in both sets of propositions, are examples of those called affirmative particular categorical propositions. The symbolic expression of either of them is the following:

$$
\text { Some } C_{4} \text { are } C_{5} \text {. }
$$

According to the preceding proposition there is at least one element of the universal set $\mathbb{U}$ considered which belongs both to $C_{4}$ and to $C_{5}$. If there is not at least one element of $\mathbb{U}$ that belongs both to $C_{4}$ and to $C_{5}$, then that proposition is false. According to section 2, any element of $\mathbb{U}$ that belongs both to $C_{4}$ and to $C_{5}$ belongs to the set which is the intersection of $C_{4}$ and $C_{5}-C_{4} \cap C_{5}$. Thus, $C_{4} \cap C_{5}$ is the set corresponding to that proposition.

The symbolic expression of any affirmative particular categorical proposition is stated again below, with the corresponding set at the right.

$$
\text { Some } C_{4} \text { are } C_{5} . \quad\left(C_{4} \cap C_{5}\right)
$$

The propositions assigned the number 4, in both sets of four propositions, are examples of those known as negative particular categorical propositions. The symbolic expression of either of them is the following:

Some $C_{4}$ are not $C_{5}$.

According to the preceding proposition, there is at least one element of the 
universal set $\mathbb{U}$ that belongs to $C_{4}$ and does not belong to $C_{5}$; that is, it belongs to $\vec{C}_{5}$. If there is not at least one element of $\mathbb{U}$ that belongs both to $C_{4}$ and to $\bar{C}_{5}$, then that proposition is false. Any element of $\mathbb{U}$ that belongs both to $C_{4}$ and to $\vec{C}_{5}$ belongs to the set that is the intersection of $C_{4}$ and $\vec{C}_{5}-C_{4} \cap \vec{C}_{5}$. Thus, $C_{4} \cap \bar{C}_{5}$ is the set corresponding to that proposition.

The symbolic expression of any particular negative categorical proposition is given below once more, with the corresponding set at the right.

$$
\text { Some } C_{4} \text { are not } C_{5} . \quad\left(C_{4} \cap \stackrel{+}{C}_{5}\right)
$$

\section{Characterization of Categorical Syllogisms}

A syllogism is a type of deductive reasoning in which a categorical proposition known as a conclusion is inferred, or deduced, from two other categorical propositions known as premises.

In this article the first premise of each categorical syllogism will be named $s_{1}$, given that "statement" or "sentence" are considered synonyms of "proposition". The second premise and the conclusion will be called $s_{2}$ and $s_{3}$, respectively. These denominations have been preferred to $q_{1}, q_{2}$ and $q_{3}$, for example, to emphasize that these are not just any three propositions, but rather three categorical propositions constituting a categorical syllogism, linked together due to their internal structures.

The predicate term of the conclusion $-s_{3}$ - is known as the major term of the syllogism and the subject term of $s_{3}$ is called the minor term of the syllogism. The major premise of the syllogism, which in this article will correspond systematically to $s_{1}$, is that which contains the major term. The minor premise of the syllogism, which in this article will correspond to $s_{2}$, is that which contains the minor term.

The third term of the syllogism does not appear in the conclusion $s_{3}$, but it does appear in each of the premises. This third term is called the middle term.

Each term of a syllogism may be assigned to a particular set. In this article the set $C_{3}$ will correspond to the major term, $C_{1}$ will correspond to the minor term, and $C_{2}$ will correspond to the middle term.

The seventh example of a categorical syllogism considered in section 7 is the following:

$s_{1}$ : All engineers are pragmatic.

$s_{2}$ : Some engineers are wealthy.

$\therefore s_{3}$ : Some wealthy persons are pragmatic.

The symbol $\therefore$ preceding $s_{3}$, means "therefore".

According to the above explanations, the sets corresponding to the different terms of that syllogism are the following: 


$$
C_{1} \text { : wealthy persons } \quad C_{2} \text { : engineers } \quad C_{3} \text { : pragmatic persons }
$$

Given these conventions, the syllogism under consideration can be presented as follows:

$s_{1}:$ All $C_{2}$ are $C_{3}$.

$s_{2}$ : Some $C_{2}$ are $C_{1}$.

$\therefore \quad s_{3}$ : Some $C_{1}$ are $C_{3}$.

\section{Specification of the Membership Table Method (MTM)}

With the terminology of propositional calculus, the most important characteristic of each categorical syllogism - that $s_{3}$ may be inferred, or deduced, from $s_{1}$ and $s_{2}$ - can be expressed as follows: $\left(s_{1} \wedge s_{2}\right) \rightarrow s_{3}$.

The internal structure of the propositions $s_{1}, s_{2}$ and $s_{3}$ is not made evident by their names. It is thus not feasible to use a resource directly from propositional calculus, such as a truth table to prove in the case of the syllogism considered that it is valid that $\left(s_{1} \wedge s_{2}\right) \rightarrow s_{3}$ is a tautology, or law, of that calculus. However, the proposition $\left(s_{1} \wedge s_{2}\right) \rightarrow s_{3}$ does make it possible to show one of the most relevant characteristics of valid categorical syllogisms: If $\left(s_{1} \wedge s_{2}\right)$ that is, the conjunction of $s_{1}$ and $s_{2}$ which is the antecedent of that proposition - is true, then $s_{3}$ (the consequent of that proposition) is true.

According to section 2, a certain set corresponds to each proposition. The sets corresponding respectively to $s_{1}, s_{2}$ and $s_{3}$ will be denominated $S_{1}, S_{2}$ and $S_{3}$. Each of those sets does reveal the relation between the terms of the corresponding proposition.

In addition, according to section 2 , the set $\left(S_{1} \cap S_{2}\right) \rightarrow S_{3}$ corresponds to the proposition $\left.s_{1} \wedge s_{2}\right) \rightarrow s_{3}$. That set will be denominated $S: S=\left(S_{1} \cap S_{2}\right) \longrightarrow S_{3}$.

According to the MTM, the corresponding set $S$ should be obtained for each categorical syllogism. If $S$ is equal to the universal set $\mathbb{U}$ - that is, if $S=\mathbb{U}$ - then that categorical syllogism is valid. If $S \neq \mathbb{U}$, then that syllogism is not valid. The different types of invalid categorical syllogisms will not be considered in this article. The MTM will be used only to determine whether the categorical syllogism considered is valid or not valid.

Recall that in the column for $S$ - the set corresponding to the syllogism considered - in the membership table of that set, the following should be examined:

a) If each element of that column is equal to one (1) then $S=\mathbb{U}$, and the syllogism considered is, therefore, valid;

b) If at least one element of that column is equal to zero (0), then $S \neq \mathbb{U}$, and the syllogism considered is, therefore, not valid. 


\section{Determining the Validity, or the Non-Validity, of Diverse Categorical Syllogisms Using the MTM: Examples}

The first six examples of the categorical syllogisms considered in this section were taken from [17].

For each example considered, the following aspects are specified: 1) the categorical syllogism expressed in natural language; 2a) that syllogism expressed in terms of the sets $C_{1}, C_{2}$ and $C_{3}$, and $2 \mathrm{~b}$ ) the corresponding sets $S_{1}, S_{2}$ and $S_{3}$, respectively; 3 ) the set $S$ corresponding to the syllogism; and 4) the membership table which makes it possible to determine whether $S=\mathbb{U}$, in which case the syllogism is valid, or whether $S \neq \mathbb{U}$, in which case the syllogism is not valid.

Example 1

$s_{1}:$ All men are mortal.

$s_{2}$ : All Greeks are men.

$\therefore s_{3}$ : All Greeks are mortal.

$C_{1}$ : Greeks $\quad C_{2}$ : men $\quad C_{3}$ : mortal beings

$$
\begin{array}{rlrl}
s_{1}: \text { All } C_{2} & \text { are } C_{3} . & S_{1} & =\left(C_{2}+C_{3}\right) \\
s_{2}: \text { All } C_{1} \text { are } C_{2} . & S_{2} & =\left(C_{1}+C_{2}\right) \\
\therefore s_{3}: \text { All } C_{1} \text { are } C_{3} . & S_{3} & =\left(C_{1}+C_{3}\right)
\end{array}
$$

$S=\left(\left(S_{1} \cap S_{2}\right) \longrightarrow S_{3}\right)=\left(\left(C_{2} \longrightarrow C_{3}\right) \cap\left(C_{1} \longrightarrow C_{2}\right)\right) \longrightarrow\left(C_{1} \longrightarrow C_{3}\right)$

\begin{tabular}{c|c|c|c|c|c|c||c}
$C_{1}$ & $C_{2}$ & $C_{3}$ & $\begin{array}{c}S_{1}= \\
C_{2}+C_{3}\end{array}$ & $\begin{array}{c}S_{2}= \\
\left(C_{1}+C_{2}\right)\end{array}$ & $\begin{array}{c}S_{3}= \\
\left(C_{1}+C_{3}\right)\end{array}$ & $\left(S_{1} \cap S_{2}\right)$ & $\begin{array}{c}S= \\
\left(S_{1} \cap S_{2}\right) \rightarrow S_{3}\end{array}$ \\
\hline 0 & 0 & 0 & 1 & 1 & 1 & 1 & 1 \\
0 & 0 & 1 & 1 & 1 & 1 & 1 & 1 \\
0 & 1 & 0 & 0 & 1 & 1 & 1 & 1 \\
0 & 1 & 1 & 1 & 1 & 1 & 1 & 1 \\
1 & 0 & 0 & 1 & 0 & 0 & 0 & 1 \\
1 & 0 & 1 & 1 & 0 & 1 & 0 & 1 \\
1 & 1 & 0 & 0 & 1 & 0 & 0 & 1 \\
1 & 1 & 1 & 1 & 1 & 1 & 1 & 1
\end{tabular}

Figure 9: Membership table of the set $S$ corresponding to the syllogism considered

The categorical syllogism considered is valid. 


\section{Example 2}

$s_{1}$ : No men are perfect.

$s_{2}$ : All Greeks are men.

$\therefore s_{3}$ : No Greeks are perfect.

$$
C_{1} \text { : Greeks } \quad C_{2} \text { : men } \quad C_{3} \text { : perfect beings }
$$

\begin{tabular}{|c|c|c|c|c|c|c|c|c|}
\hline & & & $\therefore s$ & $\begin{array}{l}: \text { No } C_{2} \text { are } \\
\text { : All } C_{1} \text { are } \\
: \text { No } C_{1} \text { are }\end{array}$ & $\begin{array}{l}C_{3} . \\
C_{2} . \\
C_{3} .\end{array}$ & $\begin{array}{l}S_{1}= \\
S_{2}= \\
S_{3}=\end{array}$ & $\begin{array}{l}\left.C_{2} \mapsto \bar{C}_{3}\right) \\
\left.C_{1} \mapsto C_{2}\right) \\
\left.C_{1} \mapsto \bar{C}_{3}\right)\end{array}$ & \\
\hline & & $=\left(\left(S_{1}\right.\right.$ & $\cap S_{2}$ & $\left.\mapsto S_{3}\right)=($ & $\left(C_{2} \longrightarrow \overleftarrow{C}_{3}\right) \cap$ & $\left.\left(C_{1} \longrightarrow C_{2}\right)\right)$ & $\left(C_{1} \longrightarrow\right.$ & \\
\hline$C_{1}$ & $C_{2}$ & $C_{3}$ & $\bar{C}_{3}$ & $\begin{array}{c}S_{1}= \\
C_{2} \mapsto \bar{C}_{3}\end{array}$ & $\begin{array}{c}S_{2}= \\
\left(C_{1} \mapsto C_{2}\right)\end{array}$ & $\begin{array}{c}S_{3}= \\
\left(C_{1}+\overleftarrow{C}_{3}\right)\end{array}$ & $\left(S_{1} \cap S_{2}\right)$ & $\begin{array}{c}S= \\
\left(S_{1} \cap S_{2}\right) \longrightarrow S_{3}\end{array}$ \\
\hline 0 & 0 & 0 & 1 & 1 & 1 & 1 & 1 & 1 \\
\hline 0 & 0 & 1 & 0 & 1 & 1 & 1 & 1 & 1 \\
\hline 0 & 1 & 0 & 1 & 1 & 1 & 1 & 1 & 1 \\
\hline 0 & 1 & 1 & 0 & 0 & 1 & 1 & 0 & 1 \\
\hline 1 & 0 & 0 & 1 & 1 & 0 & 1 & 0 & 1 \\
\hline 1 & 0 & 1 & 0 & 1 & 0 & 0 & 0 & 1 \\
\hline 1 & 1 & 0 & 1 & 1 & 1 & 1 & 1 & 1 \\
\hline 1 & 1 & 1 & 0 & 0 & 1 & 0 & 0 & 1 \\
\hline
\end{tabular}

Figure 10: Membership table of the set $S$ corresponding to the syllogism considered

The categorical syllogism considered is valid. 


\section{Example 3}

$s_{1}$ : All philosophers are wise.

$s_{2}$ : Some Greeks are philosophers.

$\therefore s_{3}$ : Some Greeks are wise.

$$
C_{1} \text { : Greeks } \quad C_{2} \text { : philosophers } \quad C_{3} \text { : wise persons }
$$

$$
\begin{array}{rlrl}
s_{1}: \text { All } C_{2} \text { are } C_{3} & S_{1} & =\left(C_{2} \mapsto C_{3}\right) \\
s_{2}: \text { Some } C_{1} \text { are } C_{2} & S_{2} & =\left(C_{1} \cap C_{2}\right) \\
\therefore s_{3}: & \text { Some } C_{1} \text { are } C_{3} & S_{3} & =\left(C_{1} \cap C_{3}\right)
\end{array}
$$$$
S=\left(\left(S_{1} \cap S_{2}\right) \longrightarrow S_{3}\right)=\left(\left(C_{2} \longrightarrow C_{3}\right) \cap\left(C_{1} \cap C_{2}\right)\right) \longrightarrow\left(C_{1} \longrightarrow C_{3}\right)
$$

\begin{tabular}{c|c|c|c|c|c|c||c}
$C_{1}$ & $C_{2}$ & $C_{3}$ & $\begin{array}{c}S_{1}= \\
C_{2}+C_{3}\end{array}$ & $\begin{array}{c}S_{2}= \\
\left(C_{1} \cap C_{2}\right)\end{array}$ & $\begin{array}{c}S_{3}= \\
\left(C_{1} \cap C_{3}\right)\end{array}$ & $\begin{array}{c}S= \\
\left(S_{1} \cap S_{2}\right)\end{array}$ \\
\hline 0 & 0 & 0 & 1 & 0 & 0 & 0 & $\left(S_{1} \cap S_{2}\right) \rightarrow S_{3}$ \\
0 & 0 & 1 & 1 & 0 & 0 & 0 & 1 \\
0 & 1 & 0 & 0 & 0 & 0 & 0 & 1 \\
0 & 1 & 1 & 1 & 0 & 0 & 0 & 1 \\
1 & 0 & 0 & 1 & 0 & 0 & 0 & 1 \\
1 & 0 & 1 & 1 & 0 & 1 & 0 & 1 \\
1 & 1 & 0 & 0 & 1 & 0 & 0 & 1 \\
1 & 1 & 1 & 1 & 1 & 1 & 1 & 1
\end{tabular}

Figure 11: Membership table of the set $S$ corresponding to the syllogism considered

The categorical syllogism considered is valid. 


\section{Example 4}

$s_{1}$ : No philosophers are wicked.

$s_{2}$ : Some Greeks are philosophers.

$\therefore s_{3}$ : Some Greeks are not wicked.

$$
C_{1} \text { : Greeks } \quad C_{2} \text { : philosophers } \quad C_{3} \text { : wicked persons }
$$

\begin{tabular}{|c|c|c|c|c|c|c|c|c|}
\hline & & & $\begin{aligned} & s_{1}: \\
& s_{2}: \\
\therefore & s_{3}:\end{aligned}$ & $\begin{array}{l}\text { No } C_{2} \text { are } C \\
\text { Some } C_{1} \text { are } \\
\text { Some } C_{1} \text { ar }\end{array}$ & $\begin{array}{l}C_{2} . \\
\text { not } C_{3} .\end{array}$ & & $\begin{array}{l}S_{1}=\left(C_{2}\right. \\
S_{2}=\left(C_{1} \cap\right. \\
S_{3}=\left(C_{1} \cap\right.\end{array}$ & $\begin{array}{l}\left.\rightarrow \bar{C}_{3}\right) \\
\left.C_{2}\right) \\
\left.\dot{C}_{3}\right)\end{array}$ \\
\hline & & $=((S$ & $\cap S_{2}$ & $\left.\mapsto S_{3}\right)=$ & $\left.C_{2} \mapsto \bar{C}_{3}\right)$ & $\left.\left(C_{1} \cap C_{2}\right)\right)$ & $\rightarrow\left(C_{1} \cap\right.$ & \\
\hline$C_{1}$ & $C_{2}$ & $C_{3}$ & $\bar{C}_{3}$ & $\begin{array}{c}S_{1}= \\
C_{2} \mapsto \bar{C}_{3}\end{array}$ & $\begin{array}{c}S_{2}= \\
\left(C_{1} \cap C_{2}\right)\end{array}$ & $\begin{array}{c}S_{3}= \\
\left(C_{1} \cap \bar{C}_{3}\right)\end{array}$ & $\left(S_{1} \cap S_{2}\right)$ & $\begin{array}{c}S= \\
\left(S_{1} \cap S_{2}\right) \mapsto S_{3}\end{array}$ \\
\hline 0 & 0 & 0 & 1 & 1 & 0 & 0 & 0 & 1 \\
\hline 0 & 0 & 1 & 0 & 1 & 0 & 0 & 0 & 1 \\
\hline 0 & 1 & 0 & 1 & 1 & 0 & 0 & 0 & 1 \\
\hline 0 & 1 & 1 & 0 & 0 & 0 & 0 & 0 & 1 \\
\hline 1 & 0 & 0 & 1 & 1 & 0 & 1 & 0 & 1 \\
\hline 1 & 0 & 1 & 0 & 1 & 0 & 0 & 0 & 1 \\
\hline 1 & 1 & 0 & 1 & 1 & 1 & 1 & 1 & 1 \\
\hline 1 & 1 & 1 & 0 & 0 & 1 & 0 & 0 & 1 \\
\hline
\end{tabular}

Figure 12: Membership table of the set $S$ corresponding to the syllogism considered

The categorical syllogism considered is valid. 


\section{Example 5}

$s_{1}:$ All Greeks are men.

$s_{2}$ : Some mortals are not men.

$\therefore s_{3}$ : Some mortals are not Greeks.

$$
C_{1} \text { : mortal beings } \quad C_{2} \text { : men } \quad C_{3} \text { : Greeks }
$$

$$
\begin{aligned}
s_{1}: \text { All } C_{3} \text { are } C_{2} . & S_{1}=\left(C_{3} \mapsto C_{2}\right) \\
s_{2}: \text { Some } C_{1} \text { are not } C_{2} . & S_{2}=\left(C_{1} \cap \vec{C}_{2}\right) \\
\therefore s_{3}: \text { Some } C_{1} \text { are not } C_{3} . & S_{3}=\left(C_{1} \cap \vec{C}_{3}\right)
\end{aligned}
$$

$S=\left(\left(S_{1} \cap S_{2}\right) \longrightarrow S_{3}\right)=\left(\left(C_{3} \longrightarrow C_{2}\right) \cap\left(C_{1} \cap \stackrel{C}{C}_{2}\right)\right) \longrightarrow\left(C_{1} \cap \overleftarrow{C}_{3}\right)$

\begin{tabular}{c|c|c|c|c|c|c|c|c||c}
$C_{1}$ & $C_{2}$ & $C_{3}$ & $\dot{C}_{2}$ & $\bar{C}_{3}$ & $\begin{array}{c}S_{1}= \\
\left.C_{3}+C_{2}\right)\end{array}$ & $\begin{array}{c}S_{2}= \\
\left(C_{1} \cap \bar{C}_{2}\right)\end{array}$ & $\begin{array}{c}S_{3}= \\
\left(C_{1} \cap \bar{C}_{3}\right)\end{array}$ & $\left(S_{1} \cap S_{2}\right)$ & $\begin{array}{c}S= \\
\left(S_{1} \cap S_{2}\right) \longrightarrow S_{3}\end{array}$ \\
\hline 0 & 0 & 0 & 1 & 1 & 1 & 0 & 0 & 0 & 1 \\
0 & 0 & 1 & 1 & 0 & 0 & 0 & 0 & 0 & 1 \\
0 & 1 & 0 & 0 & 1 & 1 & 0 & 0 & 0 & 1 \\
0 & 1 & 1 & 0 & 0 & 1 & 0 & 0 & 0 & 1 \\
1 & 0 & 0 & 1 & 1 & 1 & 1 & 1 & 1 & 1 \\
1 & 0 & 1 & 1 & 0 & 0 & 1 & 0 & 0 & 1 \\
1 & 1 & 0 & 0 & 1 & 1 & 0 & 1 & 0 & 1 \\
1 & 1 & 1 & 0 & 0 & 1 & 0 & 0 & 0 & 1
\end{tabular}

Figure 13: Membership table of the set $S$ corresponding to the syllogism considered

The categorical syllogism considered is valid. 


\section{Example 6}

$s_{1}$ : Some men are not Greeks.

$s_{2}$ : All men are mortal.

$\therefore s_{3}$ : Some mortals are not Greeks.

$$
C_{1} \text { : mortal beings } \quad C_{2} \text { : men } \quad C_{3} \text { : Greeks }
$$

$$
\begin{array}{rlrl}
s_{1}: \text { Some } C_{2} \text { are not } C_{3} . & S_{1} & =\left(C_{2} \cap \vec{C}_{3}\right) \\
s_{2}: \text { All } C_{2} \text { are } C_{1} . & S_{2}=\left(C_{2}+C_{1}\right) \\
\therefore s_{3}: \text { Some } C_{1} \text { are not } C_{3} . & S_{3}=\left(C_{1} \cap \vec{C}_{3}\right)
\end{array}
$$

$$
S=\left(\left(S_{1} \cap S_{2}\right) \longrightarrow S_{3}\right)=\left(\left(C_{2} \cap \stackrel{+}{C}_{3}\right) \cap\left(C_{2} \longrightarrow C_{1}\right)\right) \longrightarrow\left(C_{1} \cap \overleftarrow{C}_{3}\right)
$$

\begin{tabular}{c|c|c|c|c|c|c|c||c}
$C_{1}$ & $C_{2}$ & $C_{3}$ & $\vec{C}_{3}$ & $\begin{array}{c}S_{1}= \\
C_{2} \cap \bar{C}_{3}\end{array}$ & $\begin{array}{c}S_{2}= \\
\left(C_{2}+C_{1}\right)\end{array}$ & $\begin{array}{c}S_{3}= \\
\left(C_{1} \cap \bar{C}_{3}\right)\end{array}$ & $\left(S_{1} \cap S_{2}\right)$ & $\begin{array}{c}S= \\
\left(S_{1} \cap S_{2}\right)+S_{3}\end{array}$ \\
\hline 0 & 0 & 0 & 1 & 0 & 1 & 0 & 0 & 1 \\
0 & 0 & 1 & 0 & 0 & 1 & 0 & 0 & 1 \\
0 & 1 & 0 & 1 & 1 & 0 & 0 & 0 & 1 \\
0 & 1 & 1 & 0 & 0 & 0 & 0 & 0 & 1 \\
1 & 0 & 0 & 1 & 0 & 1 & 1 & 0 & 1 \\
1 & 0 & 1 & 0 & 0 & 1 & 0 & 0 & 1 \\
1 & 1 & 0 & 1 & 1 & 1 & 1 & 1 & 1 \\
1 & 1 & 1 & 0 & 0 & 1 & 0 & 0 & 1
\end{tabular}

Figure 14: Membership table of the set $S$ corresponding to the syllogism considered

The categorical syllogism considered is valid. 


\section{Example 7}

$s_{1}:$ All engineers are pragmatic.

$s_{2}$ : Some engineers are wealthy.

$\therefore s_{3}$ : Some wealthy persons are pragmatic.

$$
C_{1} \text { : wealthy persons } \quad C_{2} \text { : engineers } \quad C_{3} \text { : pragmatic persons }
$$

$$
\begin{aligned}
s_{1}: \text { All } C_{2} \text { are } C_{3} . & S_{1} & =\left(C_{2} \mapsto C_{3}\right) \\
s_{2}: \text { Some } C_{2} \text { are } C_{1} . & S_{2} & =\left(C_{2} \cap C_{1}\right) \\
\therefore s_{3}: \text { Some } C_{1} \text { are } C_{3} . & S_{3} & =\left(C_{1} \cap C_{3}\right)
\end{aligned}
$$

$S=\left(\left(S_{1} \cap S_{2}\right) \longrightarrow S_{3}\right)=\left(\left(C_{2} \longrightarrow C_{3}\right) \cap\left(C_{2} \cap C_{1}\right)\right) \longrightarrow\left(C_{1} \cap C_{3}\right)$

If it is taken into account that $\left(C_{1} \cap C_{2}\right)=\left(C_{2} \cap C_{1}\right)$, it is noted that the set corresponding to the categorical syllogism in example 7 has the same form as the set corresponding to the categorical syllogism in example 3. The latter categorical syllogism is valid. Therefore, the categorical syllogism in example 7 is valid.

\section{Example 8}

$s_{1}$ : No intellectuals are superstitious.

$s_{2}$ : Some French persons are intellectuals.

$\therefore s_{3}$ : Some French persons are not superstitious.

$C_{1}$ : French persons $\quad C_{2}$ : intellectuals $\quad C_{3}$ : superstitious persons

$$
\begin{array}{rlrl}
s_{1}: \text { No } C_{2} \text { are } C_{3} . & S_{1} & =\left(C_{2} \mapsto \stackrel{+}{C}_{3}\right) \\
s_{2}: \text { Some } C_{1} \text { are } C_{2} . & S_{2} & =\left(C_{1} \cap C_{2}\right) \\
\therefore s_{3}: \text { Some } C_{1} \text { are not } C_{3} . & S_{3}=\left(C_{1} \cap \vec{C}_{3}\right)
\end{array}
$$

$S=\left(\left(S_{1} \cap S_{2}\right) \longrightarrow S_{3}\right)=\left(\left(C_{2} \longrightarrow \bar{C}_{3}\right) \cap\left(C_{1} \cap C_{2}\right)\right) \longrightarrow\left(C_{1} \cap \vec{C}_{3}\right)$

It is noted that the set corresponding to the categorical syllogism in example 8 has the same form as the set corresponding to the categorical syllogism in example 4. The latter categorical syllogism is valid. Therefore, the categorical syllogism in example 8 is valid. 


\section{Example 9}

$s_{1}$ : All men are rational.

$s_{2}$ : All Spaniards are men.

$\therefore s_{3}$ : All Spaniards are rational.

$$
C_{1} \text { : Spaniards } \quad C_{2} \text { : men } \quad C_{3} \text { : rational men }
$$

$$
\begin{array}{rrr}
s_{1}: \text { All } C_{2} \text { are } C_{3} . & S_{1}=\left(C_{2} \mapsto C_{3}\right) \\
s_{2}: \text { All } C_{1} \text { are } C_{2} . & S_{2}=\left(C_{1} \mapsto C_{2}\right) \\
\therefore s_{3}: \text { All } C_{1} \text { are } C_{3} . & S_{3}=\left(C_{1} \mapsto C_{3}\right) \\
S=\left(\left(S_{1} \cap S_{2}\right)\right. & \left.\longrightarrow S_{3}\right)=\left(\left(C_{2} \longrightarrow C_{3}\right) \cap\left(C_{1} \longrightarrow C_{2}\right)\right) & \longrightarrow\left(C_{1} \longrightarrow C_{3}\right)
\end{array}
$$

It is noted that the set corresponding to the categorical syllogism in example 9 has the same form as the set corresponding to the categorical syllogism in example 1. The latter categorical syllogism is valid. Therefore, the categorical syllogism in example 9 is valid. 


\section{Example 10}

$s_{1}:$ All sculptors are artists.

$s_{2}$ : No artists are fossils.

$\therefore s_{3}$ : No fossils are sculptors.

$$
C_{1} \text { : fossils } \quad C_{2} \text { : artists } \quad C_{3} \text { : sculptors }
$$

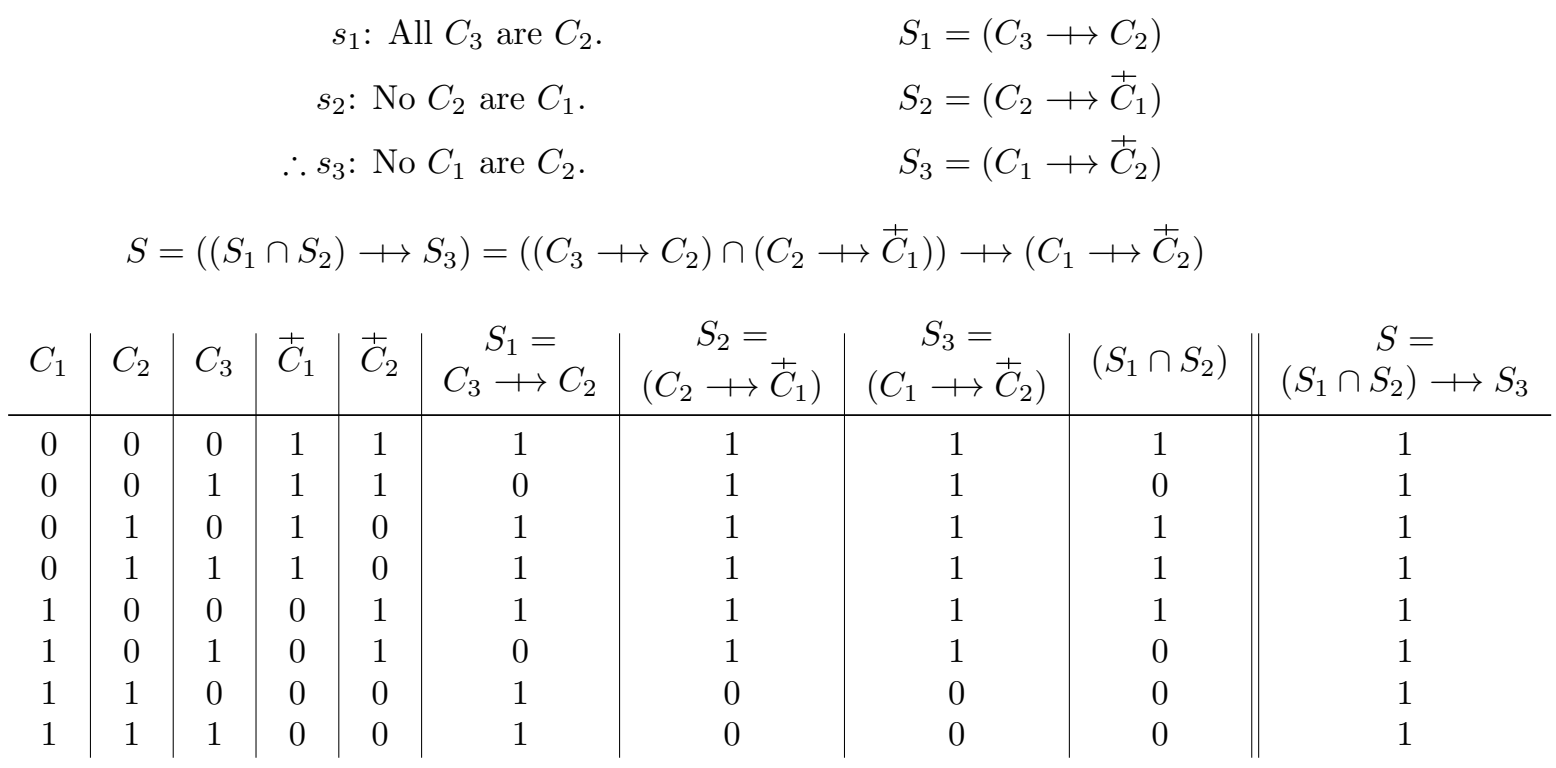

Figure 15: Membership table of the set $S$ corresponding to the syllogism considered

The categorical syllogism considered is valid. 


\section{Example 11}

$s_{1}$ : No humanists are corrupt.

$s_{2}$ : All despots are corrupt.

$\therefore s_{3}$ : No despots are humanists.

$$
C_{1} \text { : despots } \quad C_{2} \text { : corrupt persons } \quad C_{3} \text { : humanists }
$$

$$
\begin{array}{crl}
s_{1}: \text { No } C_{3} \text { are } C_{2} . & S_{1}=\left(C_{3} \mapsto \bar{C}_{2}\right) \\
s_{2}: \text { All } C_{1} \text { are } C_{2} . & S_{2}=\left(C_{1} \mapsto C_{2}\right) \\
\therefore s_{3}: \text { No } C_{1} \text { are } C_{3} . & S_{3}=\left(C_{1} \mapsto \bar{C}_{3}\right)
\end{array}
$$$$
S=\left(\left(S_{1} \cap S_{2}\right) \longrightarrow S_{3}\right)=\left(\left(C_{3} \longrightarrow \overleftarrow{C}_{2}\right) \cap\left(C_{1} \longrightarrow C_{2}\right)\right) \longrightarrow\left(C_{1} \longrightarrow \dot{C}_{3}\right)
$$

\begin{tabular}{c|c|c|c|c|c|c|c|c||c}
$C_{1}$ & $C_{2}$ & $C_{3}$ & $\bar{C}_{2}$ & $\bar{C}_{3}$ & $\begin{array}{c}S_{1}= \\
C_{3}+\bar{C}_{2}\end{array}$ & $\begin{array}{c}S_{2}= \\
\left(C_{1}+C_{2}\right)\end{array}$ & $\begin{array}{c}S_{3}= \\
\left(C_{1}+\bar{C}_{3}\right)\end{array}$ & $\left(S_{1} \cap S_{2}\right)$ & $\begin{array}{c}S= \\
\left(S_{1} \cap S_{2}\right) \longrightarrow S_{3}\end{array}$ \\
\hline 0 & 0 & 0 & 1 & 1 & 1 & 1 & 1 & 1 & 1 \\
0 & 0 & 1 & 1 & 0 & 1 & 1 & 1 & 1 & 1 \\
0 & 1 & 0 & 0 & 1 & 1 & 1 & 1 & 1 & 1 \\
0 & 1 & 1 & 0 & 0 & 0 & 1 & 1 & 0 & 1 \\
1 & 0 & 0 & 1 & 1 & 1 & 0 & 1 & 0 & 1 \\
1 & 0 & 1 & 1 & 0 & 1 & 0 & 0 & 0 & 1 \\
1 & 1 & 0 & 0 & 1 & 1 & 1 & 1 & 1 & 1 \\
1 & 1 & 1 & 0 & 0 & 0 & 1 & 0 & 0 & 1
\end{tabular}

Figure 16: Membership table of the set $S$ corresponding to the syllogism considered

The categorical syllogism considered is valid. 


\section{Example 12}

$s_{1}$ : Some mammals are dogs.

$s_{2}$ : All mammals are vertebrates.

$\therefore s_{3}$ : Some vertebrates are dogs.

$$
\begin{aligned}
& C_{1} \text { : vertebrates } \quad C_{2} \text { : mammals } \quad C_{3} \text { : dogs } \\
& s_{1} \text { : Some } C_{2} \text { are } C_{3} . \quad S_{1}=\left(C_{2} \cap C_{3}\right) \\
& s_{2} \text { : All } C_{2} \text { are } C_{1} \text {. } \quad S_{2}=\left(C_{2} \longrightarrow C_{1}\right) \\
& \therefore s_{3} \text { : Some } C_{1} \text { are } C_{3} . \quad S_{3}=\left(C_{1} \cap C_{3}\right)
\end{aligned}
$$

$S=\left(\left(S_{1} \cap S_{2}\right) \longrightarrow S_{3}\right)=\left(\left(C_{2} \cap C_{3}\right) \cap\left(C_{2} \longrightarrow C_{1}\right)\right) \longrightarrow\left(C_{1} \cap C_{3}\right)$

\begin{tabular}{c|c|c|c|c|c|c||c}
$C_{1}$ & $C_{2}$ & $C_{3}$ & $\begin{array}{c}S_{1}= \\
C_{2} \cap C_{3}\end{array}$ & $\begin{array}{c}S_{2}= \\
\left(C_{2}+C_{1}\right)\end{array}$ & $\begin{array}{c}S_{3}= \\
\left(C_{1} \cap C_{3}\right)\end{array}$ & $\begin{array}{c}S= \\
\left(S_{1} \cap S_{2}\right)\end{array}$ \\
\hline 0 & 0 & 0 & 0 & 1 & 0 & 0 & $\left(S_{1} \cap S_{2}\right)+S_{3}$ \\
0 & 0 & 1 & 0 & 1 & 0 & 0 & 1 \\
0 & 1 & 0 & 0 & 0 & 0 & 0 & 1 \\
0 & 1 & 1 & 1 & 0 & 0 & 0 & 1 \\
1 & 0 & 0 & 0 & 1 & 0 & 0 & 1 \\
1 & 0 & 1 & 0 & 1 & 1 & 0 & 1 \\
1 & 1 & 0 & 0 & 1 & 0 & 0 & 1 \\
1 & 1 & 1 & 1 & 1 & 1 & 1 & 1
\end{tabular}

Figure 17: Membership table of the set $S$ corresponding to the syllogism considered

The categorical syllogism considered is valid. 


\section{Example 13}

$s_{1}$ : No artists are Neo-Kantians.

$s_{2}$ : Some Germans are Neo-Kantians.

$\therefore s_{3}$ : Some Germans are not artists.

$$
C_{1} \text { : Germans } \quad C_{2} \text { : Neo-Kantians } \quad C_{3} \text { : artists }
$$

$$
\begin{aligned}
s_{1}: \text { No } C_{3} \text { are } C_{2} . & S_{1} & =\left(C_{3} \mapsto \vec{C}_{2}\right) \\
s_{2}: \text { Some } C_{1} \text { are } C_{2} . & S_{2} & =\left(C_{1} \cap C_{2}\right) \\
\therefore s_{3}: \text { Some } C_{1} \text { are not } C_{3} . & S_{3} & =\left(C_{1} \cap \stackrel{+}{C}_{3}\right)
\end{aligned}
$$

$S=\left(\left(S_{1} \cap S_{2}\right) \longrightarrow S_{3}\right)=\left(\left(C_{3} \mapsto \overleftarrow{C}_{2}\right) \cap\left(C_{1} \cap C_{2}\right)\right) \longrightarrow\left(C_{1} \mapsto \overleftarrow{C}_{3}\right)$

\begin{tabular}{c|c|c|c|c|c|c|c|c||c}
$C_{1}$ & $C_{2}$ & $C_{3}$ & $\bar{C}_{2}$ & $\bar{C}_{3}$ & $\begin{array}{c}S_{1}= \\
C_{3}+\bar{C}_{2}\end{array}$ & $\begin{array}{c}S_{2}= \\
\left(C_{1} \cap C_{2}\right)\end{array}$ & $\begin{array}{c}S_{3}= \\
\left(C_{1} \cap \bar{C}_{3}\right)\end{array}$ & $\left(S_{1} \cap S_{2}\right)$ & $\begin{array}{c}S= \\
\left(S_{1} \cap S_{2}\right)+S_{3}\end{array}$ \\
\hline 0 & 0 & 0 & 1 & 1 & 1 & 0 & 0 & 0 & 1 \\
0 & 0 & 1 & 1 & 0 & 1 & 0 & 0 & 0 & 1 \\
0 & 1 & 0 & 0 & 1 & 1 & 0 & 0 & 0 & 1 \\
0 & 1 & 1 & 0 & 0 & 0 & 0 & 0 & 0 & 1 \\
1 & 0 & 0 & 1 & 1 & 1 & 0 & 1 & 0 & 1 \\
1 & 0 & 1 & 1 & 0 & 1 & 0 & 0 & 0 & 1 \\
1 & 1 & 0 & 0 & 1 & 1 & 1 & 1 & 1 & 1 \\
1 & 1 & 1 & 0 & 0 & 0 & 1 & 0 & 0 & 1
\end{tabular}

Figure 18: Membership table of the set $S$ corresponding to the syllogism considered

The categorical syllogism considered is valid. 


\section{Example 14}

$s_{1}:$ All poets are visionaries.

$s_{2}$ : All prophets are visionaries.

$\therefore s_{3}$ : Some prophets are poets.

$$
C_{1} \text { : prophets } \quad C_{2} \text { : visionaries } \quad C_{3} \text { : poets }
$$

$$
\begin{aligned}
s_{1}: \text { All } C_{3} \text { are } C_{2} . & S_{1} & =\left(C_{3} \mapsto C_{2}\right) \\
s_{2}: \text { All } C_{1} \text { are } C_{2} . & S_{2} & =\left(C_{1} \mapsto C_{2}\right) \\
\therefore s_{3}: \text { Some } C_{1} \text { are } C_{3} . & S_{3} & =\left(C_{1} \cap C_{3}\right)
\end{aligned}
$$

$S=\left(\left(S_{1} \cap S_{2}\right) \longrightarrow S_{3}\right)=\left(\left(C_{3} \longrightarrow C_{2}\right) \cap\left(C_{1} \longrightarrow C_{2}\right)\right) \longrightarrow\left(C_{1} \cap C_{3}\right)$

\begin{tabular}{c|c|c|c|c|c|c||c}
$C_{1}$ & $C_{2}$ & $C_{3}$ & $\begin{array}{c}S_{1}= \\
C_{3}+C_{2}\end{array}$ & $\begin{array}{c}S_{2}= \\
\left(C_{1}+C_{2}\right)\end{array}$ & $\begin{array}{c}S_{3}= \\
\left(C_{1} \cap C_{3}\right)\end{array}$ & $\begin{array}{c}S= \\
\left(S_{1} \cap S_{2}\right)\end{array}$ & $\left(S_{1} \cap S_{2}\right)+S_{3}$ \\
\hline 0 & 0 & 0 & 1 & 1 & 0 & 1 & 0 \\
0 & 0 & 1 & 0 & 1 & 0 & 0 & 1 \\
0 & 1 & 0 & 1 & 1 & 0 & 1 & 0 \\
0 & 1 & 1 & 1 & 1 & 0 & 1 & 0 \\
1 & 0 & 0 & 1 & 0 & 0 & 0 & 1 \\
1 & 0 & 1 & 0 & 0 & 1 & 0 & 1 \\
1 & 1 & 0 & 1 & 1 & 0 & 1 & 0 \\
1 & 1 & 1 & 1 & 1 & 1 & 1 & 1
\end{tabular}

Figure 19: Membership table of the set $S$ corresponding to the syllogism considered

Given that in the column of $S$ there are membership values equal to zero (0), $S \neq \mathbb{U}$. Therefore, the categorical syllogism considered is not valid. 


\section{Example 15}

$s_{1}$ : Some landowners are not egotists.

$s_{2}$ : No philanthropists are egotists.

$\therefore s_{3}$ : Some philanthropists are landowners.

$$
C_{1} \text { : philanthropists } \quad C_{2} \text { : egotists } \quad C_{3} \text { : landowners }
$$

$$
\begin{aligned}
s_{1}: \text { Some } C_{3} \text { are not } C_{2} . & S_{1} & =\left(C_{3} \cap \bar{C}_{2}\right) \\
s_{2}: \text { No } C_{1} \text { are } C_{2} . & S_{2} & =\left(C_{1} \longrightarrow \bar{C}_{2}\right) \\
\therefore s_{3}: \text { Some } C_{1} \text { are } C_{3} . & S_{3} & =\left(C_{1} \cap C_{3}\right)
\end{aligned}
$$

$S=\left(\left(S_{1} \cap S_{2}\right) \longrightarrow S_{3}\right)=\left(\left(C_{3} \cap \stackrel{+}{C}_{2}\right) \cap\left(C_{1} \longrightarrow \stackrel{+}{C}_{2}\right)\right) \longrightarrow\left(C_{1} \cap C_{3}\right)$

\begin{tabular}{c|c|c|c|c|c|c|c||c}
$C_{1}$ & $C_{2}$ & $C_{3}$ & $\vec{C}_{2}$ & $\begin{array}{c}S_{1}= \\
C_{3} \cap \bar{C}_{2}\end{array}$ & $\begin{array}{c}S_{2}= \\
\left(C_{1}+\bar{C}_{2}\right)\end{array}$ & $\begin{array}{c}S_{3}= \\
\left(C_{1} \cap C_{3}\right)\end{array}$ & $\left(S_{1} \cap S_{2}\right)$ & $\begin{array}{c}S= \\
\left(S_{1} \cap S_{2}\right)+S_{3}\end{array}$ \\
\hline 0 & 0 & 0 & 1 & 0 & 1 & 0 & 0 & 1 \\
0 & 0 & 1 & 1 & 1 & 1 & 0 & 1 & 0 \\
0 & 1 & 0 & 0 & 0 & 1 & 0 & 0 & 1 \\
0 & 1 & 1 & 0 & 0 & 1 & 0 & 0 & 1 \\
1 & 0 & 0 & 1 & 0 & 1 & 0 & 0 & 1 \\
1 & 0 & 1 & 1 & 1 & 1 & 1 & 1 & 1 \\
1 & 1 & 0 & 0 & 0 & 0 & 0 & 0 & 1 \\
1 & 1 & 1 & 0 & 0 & 0 & 1 & 0 & 1
\end{tabular}

Figure 20: Membership table of the set $S$ corresponding to the syllogism considered

Given that in the column of $S$ there is a membership value equal to zero (0), $S \neq \mathbb{U}$. Therefore, the categorical syllogism considered is not valid. 


\section{Example 16}

$s_{1}$ : All cosmologists are scientists.

$s_{2}$ : Some scientists are polyglots.

$\therefore s_{3}$ : Some polyglots are cosmologists.

$$
C_{1} \text { : polyglots } \quad C_{2} \text { : scientists } \quad C_{3} \text { : cosmologists }
$$

$$
\begin{array}{rlrl}
s_{1}: & \text { All } C_{3} \text { are } C_{2} . & S_{1} & =\left(C_{3} \rightarrow C_{2}\right) \\
s_{2}: \text { Some } C_{2} \text { are } C_{1} . & S_{2}=\left(C_{2} \cap C_{1}\right) \\
\therefore s_{3}: \text { Some } C_{1} \text { are } C_{3} . & S_{3}=\left(C_{1} \cap C_{3}\right)
\end{array}
$$

$$
S=\left(\left(S_{1} \cap S_{2}\right) \longrightarrow S_{3}\right)=\left(\left(C_{3} \longrightarrow C_{2}\right) \cap\left(C_{2} \cap C_{1}\right)\right) \longrightarrow\left(C_{1} \cap C_{3}\right)
$$

\begin{tabular}{c|c|c|c|c|c|c||c}
$C_{1}$ & $C_{2}$ & $C_{3}$ & $\begin{array}{c}S_{1}= \\
C_{3}+C_{2}\end{array}$ & $\begin{array}{c}S_{2}= \\
\left(C_{2} \cap C_{1}\right)\end{array}$ & $\begin{array}{c}S_{3}= \\
\left(C_{1} \cap C_{3}\right)\end{array}$ & $\begin{array}{c}S= \\
\left(S_{1} \cap S_{2}\right)\end{array}$ \\
\hline 0 & 0 & 0 & 1 & 0 & 0 & 0 & $\left(S_{1} \cap S_{2}\right) \rightarrow S_{3}$ \\
0 & 0 & 1 & 0 & 0 & 0 & 0 & 1 \\
0 & 1 & 0 & 1 & 0 & 0 & 0 & 1 \\
0 & 1 & 1 & 1 & 0 & 0 & 0 & 1 \\
1 & 0 & 0 & 1 & 0 & 0 & 0 & 1 \\
1 & 0 & 1 & 0 & 0 & 1 & 0 & 1 \\
1 & 1 & 0 & 1 & 1 & 0 & 1 & 0 \\
1 & 1 & 1 & 1 & 1 & 1 & 1 & 1
\end{tabular}

Figure 21: Membership table of the set $S$ corresponding to the syllogism considered

Given that in the column of $S$ there is a membership value equal to zero (0), $S \neq \mathbb{U}$. Therefore, the categorical syllogism considered is not valid. 


\section{Discussion and Perspectives}

Reference was made to the relation existing between the truth tables of propositional calculus and the membership tables of set theory, used in the MTM, for example. Within the framework of canonical fuzzy logic (CFL) [18], the latter tables can be considered truth tables, in the strict sense. One of the main objectives of this series of articles on logic presented by the authors is to shed light on the relations existing - both in classical logic and in diverse variants of some non-classical logics - between different calculi of these logics, such as propositional calculus and predicate calculus, the last of which is expressed in the terminology of set theory. (Another of the objectives of this research program is to show how classical logic can be considered a "limit case" of those variants of non-classical logics.)

Categorical syllogisms, simple types of reasoning which has been studied a great deal, are important from historical and educational perspectives. In this research program they are a "testbed" for different methods which will be evaluated according to their possibilities of 1) being applied to determine the validity of more complex types of reasoning, and 2) being automatized for their use in disciplines such as control engineering and artificial intelligence. A method different from the MTM to determine the validity of categorical syllogisms will be presented in another article.

\section{References}

[1] Moktefi, A. \& Shin, S.-J., eds. (2013). Visual Reasoning with Diagrams. Birkhäuser/Springer.

[2] Nakatsu, R. T. (2010). Diagrammatic Reasoning in AI. Wiley.

[3] Anderson, M., B. Meyer \& Olivier, P., eds. (2002). Diagrammatic Representation and Reasoning. Springer.

[4] Sasakura, M. (2001). A Visualization Method for Knowledge Represented by General Logic Programs, Proceedings Fifth International Conference on Information Visualization (IEEE).

[5] Allwein, G. \& Barwise, J., eds. (1996). Logical Reasoning with Diagrams. Oxford University Press.

[6] Van Dyke, F. (1995). A Visual Approach to Deductive Reasoning, The Mathematics Teacher, 88, 6: 481-486, 492-494.

[7] Skliar, O., Monge, R. E., \& Gapper, S. (2015). Using Inclusion Diagrams as an Alternative to Venn Diagrams to Determine the Validity of Categorical Syllogisms. arXiv:1509.00926.

[8] Copi, I. M., Cohen, C. \& Rodych, V. (2019). Introduction to Logic, 15th ed. Routledge. 
[9] Hurley, P. J. (2015), A Concise Introduction to Logic, 12th ed. Cengage Learning.

[10] Leary, C. C. \& Kristiansen, L. (2015). A Friendly Introduction to Mathematical Logic. Milne Library.

[11] Mendelson, E. (2009). Introduction to Mathematical Logic. CRC Press.

[12] Quine, W. V. (1952; 1982). Methods of Logic, 4th ed. Harvard University Press.

[13] Goldrei, D. (2017). Classical Set Theory: For Guided Independent Study. Chapman and Hall/CRC.

[14] Cunningham, D. W. (2016). Set Theory: A First Course. Cambridge University Press.

[15] Jech, T. (2013). Set Theory. Springer.

[16] Devlin, K. (1993). The Joy of Sets: Fundamentals of Contemporary Set Theory. 2nd ed. Springer.

[17] Quine, W. V. (1952; 1982). Part II. General Terms and Quantifiers. Section 16. Syllogisms. Methods of Logic, 4th ed. Harvard University Press, p. 102.

[18] Skliar, O., Gapper, S. \& Monge, R. E. (2021). A Canonical Fuzzy Logic. arXiv:2105.12251. 